\title{
High-sensitive cardiac troponin for the diagnosis of acute myocardial infarction in different chronic kidney disease stages
}

\author{
Daijin Ren, Tianlun Huang, Xin Liu and Gaosi Xu* (0)
}

\begin{abstract}
Background: Chronic kidney disease (CKD) are associated with acute myocardial infarction (AMI). High-sensitive cardiac troponin (hs-cTn) has been evidenced to enhance the early diagnostic accuracy of AMl, but hs-cTn levels are often chronically elevated in CKD patients, which reduces their diagnostic utility. The aim of this study was to derive optimal cutoff-values of hs-cTn levels in patients with CKD and suspected AMI.
\end{abstract}

Methods: In this retrospective paper, a total of 3295 patients with chest pain (2758 in AMl group and 537 in NonAMl group) were recruited, of whom $23.1 \%$ were had an estimated glomerular filtration rate (eGFR) of $<60 \mathrm{~mL} \mathrm{~min}^{-1}$ $\left(1.73 \mathrm{~m}^{2}\right)^{-1}$. Hs-cTnl values were measured at presentation.

Results: AMl was diagnosed in $83.7 \%$ of all patients. The optimal value of hs-Tnl in diagnosing AMl was $1.15 \mathrm{ng} \mathrm{mL}^{-1}$, which were higher in males than females comparing different cutoff-values of subgroups divided by age, gender and renal function, and which increased monotonically with decreasing of eGFR because in patients with CKD without AMl, the correlation between hs-cTnl and renal function is low but significant $\left(r^{2}=0.067, P<0.001\right)$.

Conclusions: Different optimal cutoff-values of hs-cTnl in the diagnosis of AMl in patients with CKD were helpful to the clinical diagnosis of AMI in various populations and were higher in males than females, but which was needed to be validated by multicenter randomized controlled clinical studies in the future.

Keywords: Acute myocardial infarction, Chronic kidney disease, Estimated glomerular filtration rate, High-sensitivity cardiac troponin

\section{Introduction}

Chronic kidney disease (CKD) is a major public health worldwide, and the most obvious outcome of which is kidney failure requiring treatment with dialysis or transplantation at a high cost [1]. However, CKD is often associated with cardiovascular disease (CVD) [1]. It has been reported that the prevalence rate among CKD individuals encountering with acute myocardial infarction (AMI) accounted for around 73.4\%, and cardiovascular

\footnotetext{
*Correspondence: gaosixu@163.com

Department of Nephrology, The Second Affiliated Hospital of Nanchang

University, No. 1, Minde Road, Donghu District, Nanchang 330006 ,

People's Republic of China
}

mortality in dialysis patients was $10-30$ times higher than in the general population $[1,2]$. Therefore, it is crucial to quickly diagnose AMI in patients with CKD who presenting with symptoms suggestive of Acute Coronary Syndrome (ACS).

Coronary angiography (CAG) is the gold standard for diagnosing AMI, but the widespread use of contrast media (CM) may lead to a frequently overlooked complication, contrast induced-acute kidney injury (CIAKI), with rates ranging from 2.5 to $13.1 \%$ [3]. At the same time, high-sensitive cardiac troponin (hs-cTn) has been evidenced to improve the early diagnostic accuracy of AMI in many assays and has been widely applied in 
clinical practice [4], whereas elevated hs-cTn levels were observed in patients with CKD without significant myocardial necrosis, which might confuse clinicians to make an accurate and quick diagnosis when patients simultaneously presented with CKD and atypical clinical manifestation of AMI [5].

There is an urgent need to find a way to improve the diagnostic accuracy of CKD incorporating AMI instead of relying solely on CAG, and it has been attempted to improve the diagnostic accuracy of CKD with AMI by using a larger hs-cTn value to improve the diagnostic threshold, which would compensate for the loss of specificity but at the cost of decreased sensitivity [6]. In addition, the latest guide named "Fourth universal definition of myocardial infarction" has put forward that kidney disease can result in the elevation of hs-cTn, but does not mention specific data to help diagnose patients with CKD and AMI [7]. Finally, there are many other noncardiovascular conditions-related causes influences-cTn concentration in patients suspected of AMI, such as Gender, Age, Type 2 diabetes, Sampling time and etc. [8-10].

Therefore, according to different influencing factors, we conducted the current study to enhance the diagnostic confidence of AMI in patients with CKD by getting different optimal cutoffs of high-sensitive cardiac troponin I (hs-cTnI) concertation, including the largest sample size in Asia.

\section{Method and materials Study population}

Clinical characteristics and laboratory test data of the Second Affiliated Hospital of Nanchang University from January 2011 to September 2017 were collected. Patients requiring renal replacement therapy were not eligible to participate. A total of 3363 patients with suspected AMI were included for the current analysis. Hs-cTnI had not be been measured in 43 patients and estimated glomerular filtration rate (eGFR) was not available in 25 patients. The final diagnosis of AMI was determined by 2 independent cardiologists based on medical records: symptoms of myocardial ischemia, patient's present and past medical history, results of laboratory testing (including hs-TnI or serum myocardial enzyme levels), image examination, electrocardiogram (ECG) (including new ST-T changes or a new Q wave) and CAG according to 2018 "Fourth universal definition of myocardial infarction" [7], as shown in Fig. 1.

\section{Clinical and laboratory examination}

All the detailed clinical history, including age, sex, risk factors (hypertension, hypercholesterolemia, diabetes mellitus (DM), current smoking and history of smoking), coronary artery disease (CAD) history, vital status,

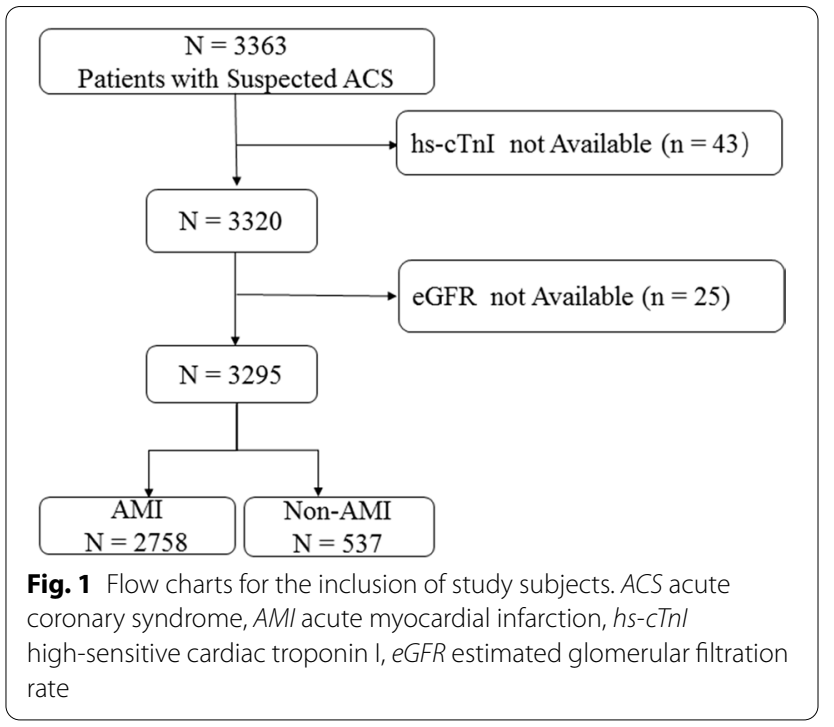

laboratory measurements (serum creatinine, hs-cTnI) and time from symptom onset, was extracted from medical recordings.

Blood and vital status were collected directly at presentation. The hs-cTnI assay (ARCHITECT STAT hs Troponin I, Abbott Diagnostics, Abbott Park, IL) that was drawn in the medical records has an assay range of $0-50,000 \mathrm{ng} \mathrm{L}^{-1}$, with the detection limit of $1.9 \mathrm{ng} \mathrm{L}^{-1}$ and the 99th-percentile diagnostic cutoff of $30 \mathrm{ng} \mathrm{L}^{-1}$ [11]. DM was defined in our hospital in accordance with OGTT-based WHO criteria [12], and hypertension was diagnosed as systolic blood pressure (SBP) of $140 \mathrm{mmHg}$ or above, or diastolic blood pressure (DBP) of $90 \mathrm{mmHg}$ or above, or taking hypotensive drugs [13]. Estimated glomerular filtration rate (eGFR) was computed with CKD-EPI creatinine equation in accordance with 2012 KDIGO guidelines [14].

\section{Statistical methods}

Continuous variables are expressed as medians (1st quartile, 3rd quartile) and categorical variables as numbers and percentages. normally distributed variables were compared with Student's t-test and skewed distribution variables were analyzed with the Mann-Whitney $\mathrm{U}$ test, and categorical variables were assessed by the chi-square test. Receiver operating characteristic (ROC) curves were constructed to calculate the cutoff-value of hs-TnI to diagnose AMI, in order to improve diagnostic accuracy of hs-cTnI measured at presentation in patients with suspected AMI. Diagnostic accuracy was quantified by the area under the curve (AUC), plotted by MedCalc statistical software. Correlation between hs-cTnI and renal function was quantified by Pearson's correlation 
coefficient, constructed by GraphPad Prism version 8.0. Receiver Other statistical analysis was performed with IBM SPSS Statistics for Windows, version 23.0 (Chicago, IL, USA). $P$ values $<0.05$ were considered statistically significant.

\section{Results}

\section{Patient characteristics}

In the study, 3295 patients suspected with AMI were recruited (2758 in AMI group and 537 in Non-AMI group). The baseline characteristics of patients in each group are shown in Table 1. Mean age of the patients was 61 years and $2483(75.4 \%)$ of the participants were male. Patients in AMI group were older with higher proportions of males, hypertension, hypercholesterolemia, diabetes mellitus, family history of CAD and prior CAD than Non-AMI group $(P<0.001)$. Patients of AMI group had higher hs-TnI level compared with Non-AMI group $(P<0.001)$ and baseline hs-TnI concentrations were 20.91 $(2.46,48.03) \mathrm{ng} \mathrm{mL} L^{-1}$ and $0.57(0.16,0.44) \mathrm{ng} \mathrm{mL}{ }^{-1}$, respectively. There was no significant influence in current smoking or history of smoking between two groups, $P$ values of which were 0.069 and 0.076 , respectively. There was also no difference in time from symptom onset between two groups.

\section{Hs-cTnl and renal function}

We assessed the association of hs-cTnI with measures of renal function in patients with CKD [eGFR $<60 \mathrm{~mL} \mathrm{~min}^{-1}$ $\left(1.73 \mathrm{~m}^{2}\right)^{-1}$ ] by Pearson's correlation coefficient, and compared hs-cTnI levels between patients with or without AMI (as shown in Fig. 2). There was a low but significant correlation between hs-cTnI and renal function in CKD patients without AMI $\left(\mathrm{r}^{2}=0.067, P<0.001\right)$, but there was no correlation in CKD patients with AMI $\left(r^{2}=0.003, P=0.23\right)$. Furthermore, we found that CKD could result in the elevation of hs-cTnI without AMI.

Based on the above, we concluded that in presenting with CKD and non-AMI patients, the lower the eGFR, the higher the hs-cTnI concentration, but in AMI individuals, which was not.

\section{Diagnostic accuracy of hs-cTnl at presentation}

By constructing ROC curve, we got the optimal cutoffvalue for diagnosis of AMI in all suspicious patients, which appeared to be an hs-TnI level of $1.15 \mathrm{ng} \mathrm{mL}{ }^{-1}$ with $82.5 \%$ sensitivity and $95.4 \%$ specificity. AUC was 0.924 [95\% CI $(0.914,0.933)$ ] (Fig. 3). Then, we grouped those patients by different age, sex, and eGFR categories (Table 2 and Fig. 4), and calculated their optimal

Table 1 Baseline characteristics of patients

\begin{tabular}{|c|c|c|c|c|}
\hline & Total & AMI & Non-AMI & $P$ value \\
\hline Number of patients $(n, \%)$ & 3295 & $2758(83.7)$ & $537(16.3)$ & \\
\hline Age (years) & $61(48,71)$ & $64(55,74)$ & $57(43,70)$ & $<0.001$ \\
\hline Sex (male) & $2483(75.4)$ & $2165(78.5)$ & $318(59.2)$ & $<0.001$ \\
\hline \multicolumn{5}{|l|}{ Risk factors } \\
\hline Hypertension & $1688(51.2)$ & $1567(56.8)$ & $121(22.5)$ & $<0.001$ \\
\hline Hypercholesterolemia & $930(28.2)$ & $880(31.9)$ & $50(9.3)$ & $<0.001$ \\
\hline Diabetes mellitus & $682(20.7)$ & $646(23.4)$ & $26(4.8)$ & $<0.001$ \\
\hline Current smoking & $1519(46.1)$ & $1288(46.7)$ & $231(43.2)$ & 0.069 \\
\hline History of smoking & $1770(53.7)$ & $1497(54.3)$ & $273(50.8)$ & 0.076 \\
\hline \multicolumn{5}{|l|}{ History } \\
\hline Family history of $C A D$ & $82(2.6)$ & $77(2.8)$ & $5(0.87)$ & $<0.001$ \\
\hline Prior CAD & $758(23.0)$ & $723(26.2)$ & $35(6.5)$ & $<0.001$ \\
\hline \multicolumn{5}{|l|}{ Vital status } \\
\hline Heart rate (bpm) & $76(64,88)$ & $79(66,94)$ & $74(63,84)$ & $<0.001$ \\
\hline Systolic blood pressure (mmHg) & $125(115,137)$ & $143(129,162)$ & $119(114,132)$ & $<0.001$ \\
\hline Diastolic blood pressure $(\mathrm{mmHg})$ & $81(71,93)$ & $85(76,94)$ & $73(68,82)$ & $<0.001$ \\
\hline Hs-cTnl (ng mL ${ }^{-1}$ ) & $19.43(1.38,48.60)$ & $20.91(2.46,48.03)$ & $0.57(0.16,0.44)$ & $<0.001$ \\
\hline \multicolumn{5}{|l|}{ Time from symptom onset (h) } \\
\hline$<6(\mathrm{n}, \%)$ & $1267(38.5)$ & $1063(38.5)$ & $204(38.0)$ & 0.977 \\
\hline$\geq 6(n, \%)$ & $2028(61.5)$ & $1695(61.5)$ & $333(62.0)$ & 0.976 \\
\hline
\end{tabular}

For group comparisons, Student's $t$ test was used for normally distributed variables and non-parametric Mann-Whitney tests for non-normally distributed variables. Categorical variables were compared with a $x^{2}$-test

$A M I$ acute myocardial infarction, $C A D$ coronary artery disease, $H s-c T n /$ high-sensitive cardiac troponin I 


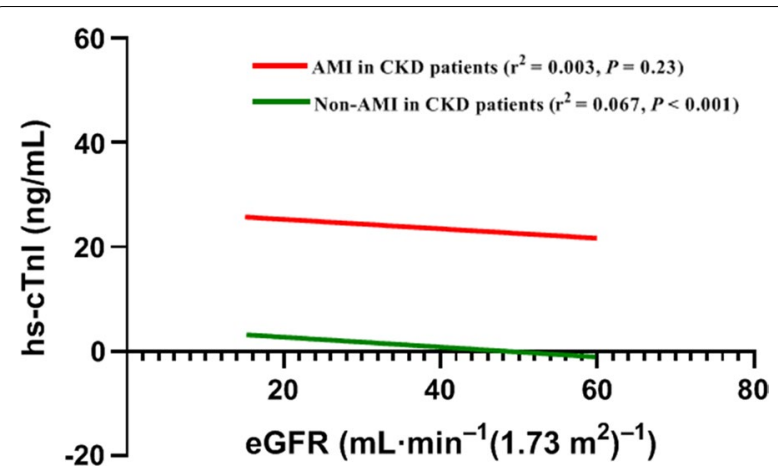

Fig. 2 The association of hs-cTnl with measures of renal function

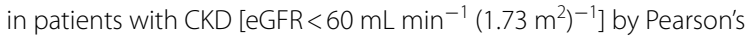
correlation coefficient, and compared hs-cTnl levels between patients with or without AMI. There was a low but significant correlation between hs-CTnl and renal function in CKD patients without AMl $\left(r^{2}=0.067, P<0.001\right)$, but there was no correlation in CKD patients with AMI $\left(r^{2}=0.003, P=0.23\right)$. Furthermore, we found that $C K D$ could result in the elevation of hs-cTnl without AMI. AMl acute myocardial infarction, eGFR estimated glomerular filtration rate, $h s-c T n l$ high-sensitive cardiac troponin I, CKD chronic kidney disease

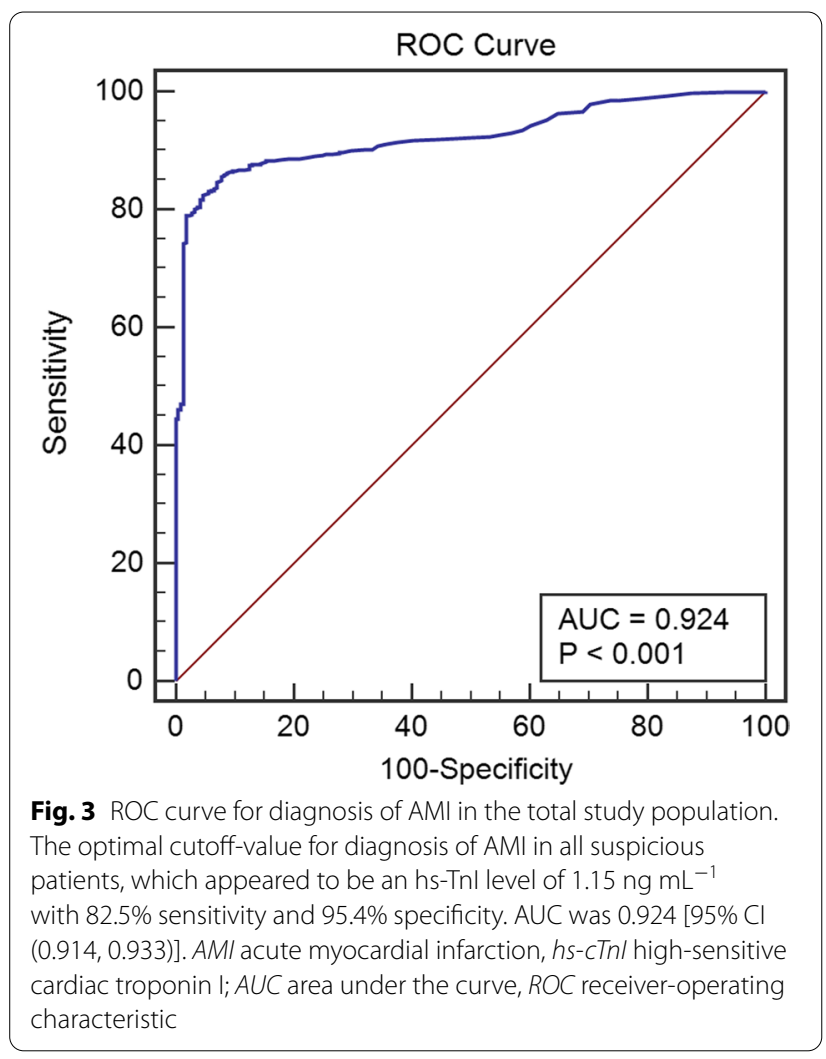

cutoff-values of hs-TnI levels to improve diagnostic accuracy of $A M I$ in CKD patients, respectively.

In the "Male Patients younger than 60 years with eGFR $\geq 60 \mathrm{~mL} \mathrm{m^{-1 }}\left(1.73 \mathrm{~m}^{2}\right)^{-1 "}$ group, there were
896 patients. AMI was the adjudicated final diagnosis in $89.1 \%$ of them. The optimal cutoff-value for diagnosis of AMI was $0.24 \mathrm{ng} \mathrm{mL}^{-1}$ with $91.6 \%$ sensitivity and $100.0 \%$ specificity. AUC was 0.984 [ $95 \%$ CI $(0.973,0.991)]$ (Fig. 4a).

In the "Male Patients aged 60 or above with

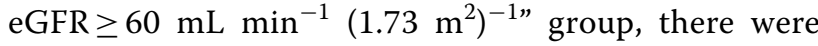
1088 patients. AMI was the adjudicated final diagnosis in $94.5 \%$ of them. The optimal cutoff-value for diagnosis of AMI was $0.31 \mathrm{ng} \mathrm{mL}^{-1}$ with $90.9 \%$ sensitivity and $100.0 \%$ specificity. AUC was 0.962 [95\% CI (0.949, 0.973)] (Fig. 4b).

In the "Female Patients younger than 60 years with eGFR $\geq 60 \mathrm{~mL} \mathrm{~min}{ }^{-1}\left(1.73 \mathrm{~m}^{2}\right)^{-1}$ " group, there were 154 patients. AMI was the adjudicated final diagnosis in $52.6 \%$ of them. The optimal cutoff-value for diagnosis of AMI was $0.27 \mathrm{ng} \mathrm{mL}^{-1}$ with $93.2 \%$ sensitivity and $100.0 \%$ specificity. AUC was 0.979 [95\% CI (0.941, 0.995)] (Fig. 4c).

In the "Female Patients aged 60 or above with eGFR $\geq 60 \mathrm{~mL} \min ^{-1}\left(1.73 \mathrm{~m}^{2}\right)^{-1}$ " group, there were 396 patients. AMI was the adjudicated final diagnosis in $90.2 \%$ of them. The optimal cutoff-value for diagnosis of AMI was $0.27 \mathrm{ng} \mathrm{mL}^{-1}$ with $91.0 \%$ sensitivity and $100.0 \%$ specificity. AUC was 0.946 [95\% CI (0.918, 0.966)] (Fig. 4d).

In the "Male Patients younger than 60 years with $30 \leq \mathrm{eGFR}<60 \mathrm{~mL} \mathrm{~min}^{-1}\left(1.73 \mathrm{~m}^{2}\right)^{-1}$ " group, there were 76 patients. AMI was the adjudicated final diagnosis in $73.7 \%$ of them. The optimal cutoff-value for diagnosis of AMI was $0.72 \mathrm{ng} \mathrm{mL}^{-1}$ with $81.4 \%$ sensitivity and $100.0 \%$ specificity. AUC was 0.869 [95\% CI (0.772, 0.936)] (Fig. 4e).

In the "Male Patients aged 60 or above with $30 \leq \mathrm{eGFR}<60 \mathrm{~mL} \min ^{-1}\left(1.73 \mathrm{~m}^{2}\right)^{-1}$ " group, there were 330 patients. AMI was the adjudicated final diagnosis in $80.9 \%$ of them. The optimal cutoff-value for diagnosis of AMI was $0.50 \mathrm{ng} \mathrm{mL}^{-1}$ with $89.5 \%$ sensitivity and $100.0 \%$ specificity. AUC was 0.959 [95\% CI (0.932, 0.978)] (Fig. 4f).

In the "Female Patients aged 60 or above with $30 \leq \mathrm{eGFR}<60 \mathrm{~mL} \min ^{-1}\left(1.73 \mathrm{~m}^{2}\right)^{-1}$ " group, there were 178 patients. AMI was the adjudicated final diagnosis in $71.9 \%$ of them. The optimal cutoff-value for diagnosis of AMI was $0.59 \mathrm{ng} \mathrm{mL}^{-1}$ with $91.4 \%$ sensitivity and $100.0 \%$ specificity. AUC was 0.952 [95\% CI (0.910, 0.979)] (Fig. 4g).

In the "Male Patients aged 60 or above with $15 \leq \mathrm{eGFR}<30 \mathrm{~mL} \min ^{-1}\left(1.73 \mathrm{~m}^{2}\right)^{-1}$ " group, there were 89 patients. AMI was the adjudicated final diagnosis in $57.3 \%$ of them. The optimal cutoff-value for diagnosis of AMI was $2.61 \mathrm{ng} \mathrm{mL}^{-1}$ with $80.4 \%$ sensitivity 
Table 2 Diagnostic accuracy of hs-cTnl for AMI in patients according to sex, age and eGFR category

\begin{tabular}{|c|c|c|c|c|c|}
\hline & AMI $(n, \%)$ & Sensitivity $(\%, 95 \% \mathrm{CI})$ & Specificity (\%, 95\% CI) & AUC $(95 \% \mathrm{Cl})$ & $P$ value \\
\hline All patients $(n=3295)$ & $2758(83.7)$ & & & & \\
\hline Cut-off $=1.15 \mathrm{ng} \mathrm{mL}^{-1}$ & & $82.5(81.0,83.9)$ & $95.4(91.7,97.8)$ & $0.924(0.914,0.933)$ & $<0.001$ \\
\hline $\begin{array}{l}\text { (a) Male patients younger than } 60 \text { years with } \\
\text { eGFR } \geq 60 \mathrm{~mL} \mathrm{~min}^{-1}\left(1.73 \mathrm{~m}^{2}\right)^{-1}(n=896)\end{array}$ & $798(89.1)$ & & & & \\
\hline Cut-off $=0.24 \mathrm{ng} \mathrm{mL}^{-1}$ & & $91.6(89.5,93.4)$ & $100.0(96.3,100.0)$ & $0.984(0.973,0.991)$ & $<0.001$ \\
\hline 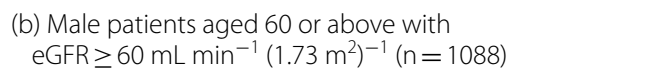 & $1028(94.5)$ & & & & \\
\hline Cut-off $=0.31 \mathrm{ng} \mathrm{mL}^{-1}$ & & $90.9(88.9,92.6)$ & $100.0(95.9,100.0)$ & $0.962(0.949,0.973)$ & $<0.001$ \\
\hline $\begin{array}{l}\text { (c) Female patients younger than } 60 \text { years with } \\
\text { eGFR } \geq 60 \mathrm{~mL} \mathrm{~min}{ }^{-1}\left(1.73 \mathrm{~m}^{2}\right)^{-1}(\mathrm{n}=154)\end{array}$ & $81(52.6)$ & & & & \\
\hline Cut-off $=0.27 \mathrm{ng} \mathrm{mL}^{-1}$ & & $93.2(84.9,97.8)$ & $100.0(95.5,100.0)$ & $0.979(0.941,0.995)$ & $<0.001$ \\
\hline $\begin{array}{l}\text { (d) Female patients aged } 60 \text { or above with } \\
\text { GFR } \geq 60 \mathrm{~mL} \mathrm{~min}^{-1}\left(1.73 \mathrm{~m}^{2}\right)^{-1}(\mathrm{n}=396)\end{array}$ & $357(90.2)$ & & & & \\
\hline Cut-off $=0.27 \mathrm{ng} \mathrm{mL}^{-1}$ & & $91.0(87.5,93.8)$ & $100.0(93.0,100.0)$ & $0.946(0.918,0.966)$ & $<0.001$ \\
\hline $\begin{array}{l}\text { (e) Male patients younger than } 60 \text { years with } \\
30 \leq \mathrm{eGFR<60 \textrm {mL } \mathrm { min }}{ }^{-1}\left(1.73 \mathrm{~m}^{2}\right)^{-1}(n=76)\end{array}$ & $56(73.7)$ & & & & \\
\hline Cut-off $=0.72 \mathrm{ng} \mathrm{mL}^{-1}$ & & $81.4(66.6,91.6)$ & $100.0(89.4,100.0)$ & $0.869(0.772,0.936)$ & $<0.001$ \\
\hline $\begin{array}{l}\text { (f) Male patients aged } 60 \text { or above with } \\
30 \leq \mathrm{eGFR}<60 \mathrm{~mL} \mathrm{~min}^{-1}\left(1.73 \mathrm{~m}^{2}\right)^{-1}(n=330)\end{array}$ & $267(80.9)$ & & & & \\
\hline \multicolumn{2}{|l|}{ Cut-off $=0.50 \mathrm{ng} \mathrm{mL}^{-1}$} & $89.5(85.2,92.9)$ & $100.0(94.3,100.0)$ & $0.959(0.932,0.978)$ & $<0.001$ \\
\hline \multicolumn{6}{|c|}{ 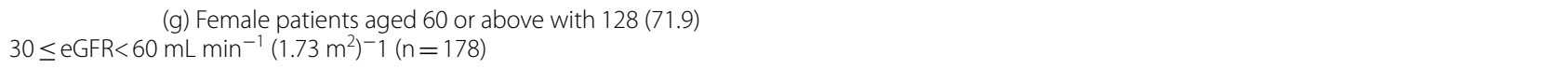 } \\
\hline \multicolumn{2}{|l|}{ Cut-off $=0.59 \mathrm{ng} \mathrm{mL}^{-1}$} & $91.4(85.1,95.6)$ & $100.0(92.9,100.0)$ & $0.952(0.910,0.979)$ & $<0.001$ \\
\hline 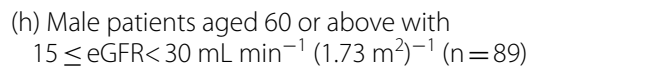 & $51(57.3)$ & & & & \\
\hline Cut-off $=2.61 \mathrm{ng} \mathrm{mL}^{-1}$ & & $80.4(66.9,90.2)$ & $92.1(78.6,98.3)$ & $0.907(0.827,0.958)$ & $<0.001$ \\
\hline 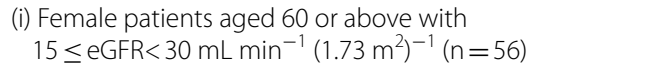 & $28(50.0)$ & & & & \\
\hline Cut-off $=1.90 \mathrm{ng} \mathrm{mL}^{-1}$ & & $89.3(71.8,97.7)$ & $100.0(87.7,100.0)$ & $0.943(0.846,0.987)$ & $<0.001$ \\
\hline
\end{tabular}

AMI acute myocardial infarction, eGFR estimated glomerular filtration rate, $h s$-cThl high-sensitive cardiac troponin I, $C l$ confidence interval, $A U C$ area under the curve

and $92.1 \%$ specificity. AUC was 0.907 [95\% CI $(0.827$, 0.958)] (Fig. 4h).

In the "Female Patients aged 60 or above with

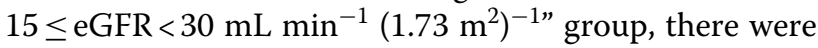
56 patients. AMI was the adjudicated final diagnosis in $50.0 \%$ of them. The optimal cutoff-value for diagnosis of AMI was $1.90 \mathrm{ng} \mathrm{mL}^{-1}$ with $89.3 \%$ sensitivity and $100.0 \%$ specificity. AUC was 0.943 [95\% CI $(0.846,0.987)]$ (Fig. 4i).

However, in the "Female Patients younger than 60 years

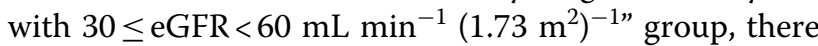
were only 18 patients and 4 patients were diagnosed as AMI, hs-cTnI data of which was $10.04(0.28,11.36)$ $\mathrm{ng} \mathrm{mL} \mathrm{m}^{-1}$. In the "Male Patients younger than 60 years with $15 \leq \mathrm{eGFR}<30 \mathrm{~mL} \min ^{-1}\left(1.73 \mathrm{~m}^{2}\right)^{-1}$ " group, there were only 9 patients and 5 patients were diagnosed as AMI, hs-cTnI data of which was $8.88(0.90,2.60)$

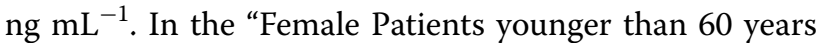
with $15 \leq \mathrm{eGFR}<30 \mathrm{~mL} \mathrm{~min}^{-1}\left(1.73 \mathrm{~m}^{2}\right)^{-1}$ " group, there were only 5 patients and 1 patients were diagnosed as AMI, hs-cTnI data of which was $0.49(0.46,0.51)$ ng $\mathrm{mL}^{-1}$. Considering these groups with small sample sizes, we did not perform ROC curve with them.

\section{Discussion}

Patients with CKD are often associated with CVD, especially, AMI with atypical symptoms [15], who often do not receive the best possible treatment for AMI, including antithrombotic drugs and coronary angiography. It was hard for clinicians to make the correct diagnosis of AMI because patients with CKD were more insensitive to chest pain and hs-cTnI levels often rise chronically even in the absence of AMI [16, 17]. And the latest universal definition of AMI also referred that many patients with chronic kidney disease (CKD) have elevation of hs-cTnI values [7], but which did not provide for optimal cutoffs of it. Therefore, to improve the diagnostic accuracy of AMI in patients with CKD, it is necessary to calculate cutoffs of hs-cTnI levels.

Some studies had tested this cutoff value in patients with different renal functions [10, 18]. A prospective multicenter diagnostic study enrolling individuals with 

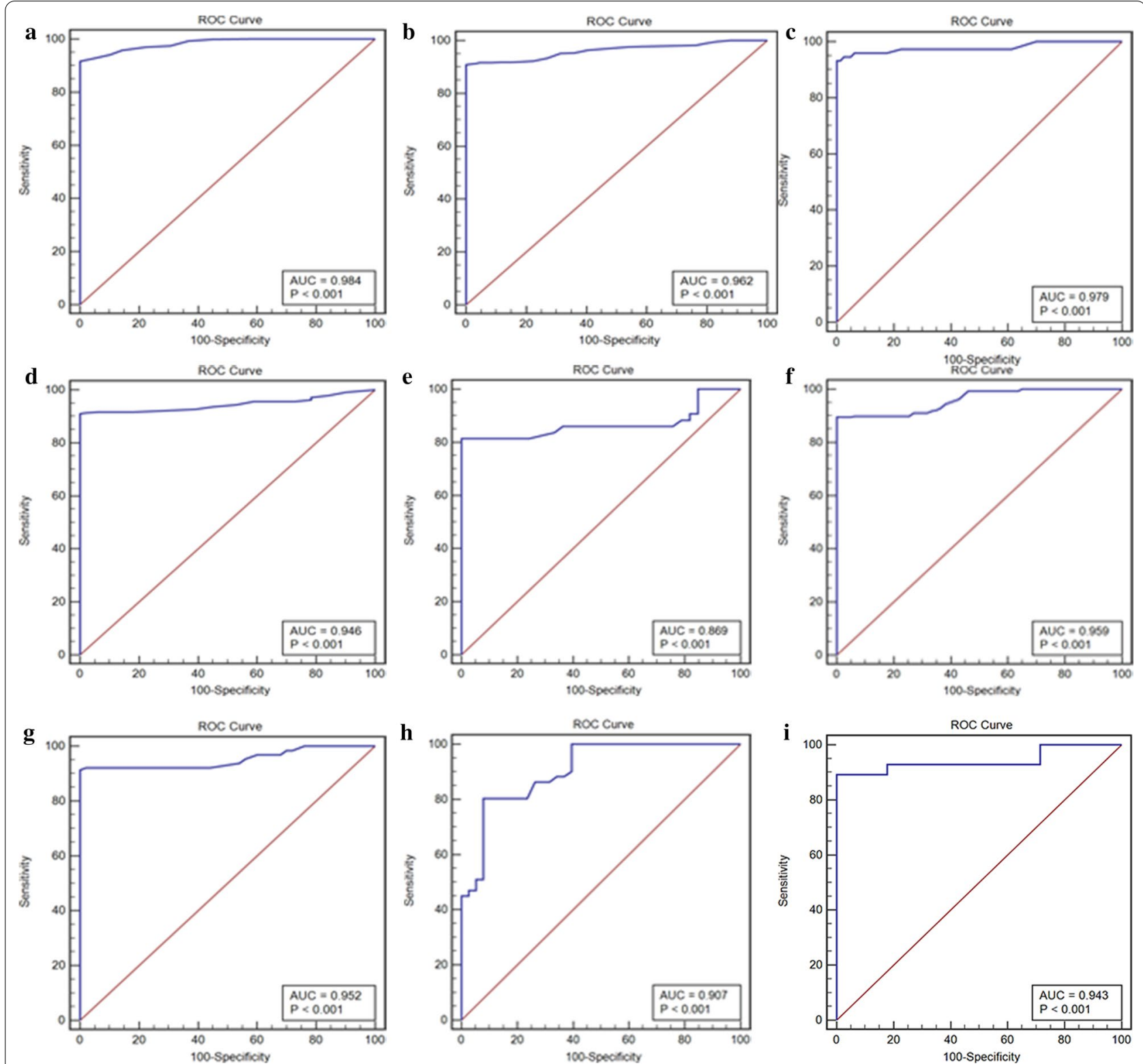

Fig. 4 ROC curve for diagnosis of AMI divided by age, gender and renal function. a Male patients younger than 60 years with eGFR $\geq 60 \mathrm{~mL} \mathrm{~min}{ }^{-1}$ $\left(1.73 \mathrm{~m}^{2}\right)^{-1}$, Cut-off $=0.24 \mathrm{ng} \mathrm{mL}^{-1}$ with $91.6 \%$ sensitivity and $100.0 \%$ specificity. AUC $=0.984[95 \% \mathrm{Cl}(0.973,0.991)]$. b Male patients aged 60 or above with eGFR $\geq 60 \mathrm{~mL} \mathrm{~min}{ }^{-1}\left(1.73 \mathrm{~m}^{2}\right)^{-1}$, Cut-off $=0.31 \mathrm{ng} \mathrm{mL}^{-1}$ with $90.9 \%$ sensitivity and $100.0 \%$ specificity. AUC $=0.962$ [95\% Cl $(0.949,0.973)]$. c Female patients younger than 60 years with eGFR $\geq 60 \mathrm{~mL} \mathrm{~min}^{-1}\left(1.73 \mathrm{~m}^{2}\right)^{-1}$, Cut-off $=0.27 \mathrm{ng} \mathrm{mL}^{-1}$ with $93.2 \%$ sensitivity and $100.0 \%$ specificity. $A U C=0.979[95 \% \mathrm{Cl}(0.941,0.995)]$. $\mathbf{d}$ Female patients aged 60 or above with eGFR $\geq 60 \mathrm{~mL} \mathrm{~min}^{-1}\left(1.73 \mathrm{~m}^{2}\right)^{-1}$, Cut-off $=0.27 \mathrm{ng} \mathrm{mL}^{-1}$ with $91.0 \%$ sensitivity and $100.0 \%$ specificity. AUC $=0.946[95 \% \mathrm{Cl}(0.918,0.966)]$. e Male patients younger than 60 years with $30 \leq$ eGFR $<60 \mathrm{~mL} \mathrm{~min}{ }^{-1} \cdot\left(1.73 \mathrm{~m}^{2}\right)^{-1}$, Cut-off $=0.72 \mathrm{ng} \mathrm{mL}^{-1}$ with $81.4 \%$ sensitivity and $100.0 \%$ specificity. AUC $=0.869[95 \% \mathrm{Cl}(0.772,0.936)]$. f Male patients aged 60 or above with $30 \leq \mathrm{eGFR}<60 \mathrm{~mL} \mathrm{~min}^{-1} \cdot\left(1.73 \mathrm{~m}^{2}\right)^{-1}$, Cut-off $=0.50 \mathrm{ng} \mathrm{mL}^{-1}$ with $89.5 \%$ sensitivity and $100.0 \%$ specificity. $A U C=0.959[95 \% \mathrm{Cl}(0.932,0.978)]$. g Female patients aged 60 or above with $30 \leq \mathrm{eGFR}<60 \mathrm{~mL} \mathrm{~min}{ }^{-1} \cdot\left(1.73 \mathrm{~m}^{2}\right)^{-1}$, Cut-off $=0.59 \mathrm{ng} \mathrm{mL} \mathrm{mith}^{-1}$ with 91.4\% sensitivity and $100.0 \%$ specificity. $\mathrm{AUC}=0.952[95 \% \mathrm{Cl}(0.910,0.979)]$. h Male patients aged 60 or above with $15 \leq \mathrm{eGFR}<30 \mathrm{~mL} \mathrm{~min}^{-1}(1.73$ $\left.\mathrm{m}^{2}\right)^{-1}$, Cut-off $=2.61 \mathrm{ng} \mathrm{mL}^{-1}$ with $80.4 \%$ sensitivity and $92.1 \%$ specificity. $\mathrm{AUC}=0.907$ [95\% Cl $\left.(0.827,0.958)\right]$. i Female Patients aged 60 or above with $15 \leq \mathrm{eGFR}<30 \mathrm{~mL} \mathrm{~min}{ }^{-1}\left(1.73 \mathrm{~m}^{2}\right)^{-1}$, Cut-off $=1.90 \mathrm{ng} \mathrm{mL}^{-1}$ with $89.3 \%$ sensitivity and $92.1 \%$ specificity. AUC $=0.943$ [95\% Cl $\left.(0.846,0.987)\right]$. AMI acute myocardial infarction, $h s-C T n /$ high-sensitive cardiac troponin I, AUC area under the curve, ROC receiver-operating characteristic, eGFR estimated glomerular filtration rate 
suspected non-ST-segment elevation myocardial infarction, might better help diagnose atypical symptoms of AMI by computing different cut-off values of hs-cTnI and raising the 0/1-h algorithm, but which with high sensitivity and lower specificity easily leading to misdiagnosis, and which was unrealistic to observe troponin changes dynamically by repeated blood, because once the suspected patient went to the hospital, the coronary angiography should be performed immediately to assist the diagnosis, so that the troponin cannot be taken again and patients with renal insufficiency were more likely to be complicated with anemia so that repeated blood collection might aggravate the anemia [10]. In addition, another similar study also showed the above shortcomings by calculating different cut-off values of hs-cTnI and raising the 0/3-h algorithm [18]. Furthermore, two retrospective studies computed different cut-off values of hs-cTn levels according to eGFR category, which also presenting with high sensitivity and lower specificity $[4,19]$, but there were other factors influencing hs-cTnI concentrations as well as the triage of patients with suspected AMI. Some studies indicated that age was the most important non-cardiac determinant of hs-cTnI levels [20,21], and patients aged over 60 years had higher hs-cTnI levels [22]. Gender was also an important factor and the median hs-cTnI concentration was significantly lower in women. It has been suggested that the underdiagnosis of AMI in women was due to the inappropriate diagnostic thresholds and gender-specific thresholds were recommended for hs-cTnI assays to improve the diagnostic accuracy [23].

In this retrospective study involving 3295 patients,

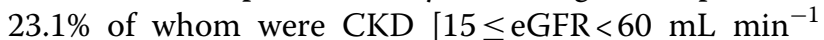
$\left(1.73 \mathrm{~m}^{2}\right)^{-1}$ ] excluding patients of end-stage kidney disease, constructed the diagnostic performance of an hs$\mathrm{cTnI}$ assay taken at presentation in patients presenting with acute chest pain. We compared hs-TnI levels in AMI and non-AMI patients, as well as in the subgroups of different age, sex and renal function categories. And we made a simple linear regression analysis to simulate the association of hs-cTnI with renal function. Further, we performed ROC curves to compute optimal hs-TnI levels for helping diagnose of AMI in different categories individuals.

To our acknowledgment, our study is the first analysis to calculate optimal cutoff-values to improve the diagnostic accuracy of AMI in patients with CKD, based on different age, gender and renal kidney subgroups, which may promote early diagnosis and intervention of AMI further to improve prognosis in CKD patients.

Our study has shown that the correlation of hs-cTnI with eGFR are weak but significant in CKD patients $\left[15 \leq \mathrm{eGFR}<60 \mathrm{~mL} \min ^{-1}\left(1.73 \mathrm{~m}^{2}\right)^{-1}\right]$ without AMI patients. That is to say, the cutoff-values of hs-cTnI concentrations were increased monotonically with decreasing of eGFR, but in CKD with AMI individuals, which were not. This outcome was in accordance with the literature, which has been made an explanation that CKD patients were more insensitive to chest pain, leading to missed diagnosis of symptomLess AMI patients with CKD [19], on the other hand, we also made another explanation, which was many patients diagnosed with AMI have reached the highest concentration of hscTnI $\left(50 \mathrm{ng} \mathrm{mL}^{-1}\right.$ ) that could be detected in our laboratory, at the same time, hs-cTnI levels were independent of changes in eGFR. And we performed 9 ROC curves based on subgroups divided by age, gender and eGFR to get different cutoff-values of hs-cTnI concentrations, and 7 of these groups' specificity reached to $100 \%$, which were significantly higher than other similar studies $[4,10,18$, 19]. Our data also showed hs-cTnI concentrations were higher in males than females, comparing different genders in the same age and kidney function group, which was in agreement with the literature [22]. Further, the cutoff-value of hs-cTnI concentrations in patients aged 60 or above was not higher than below 60 years, comparing different age stages in the same gender and kidney function group in disagreement with the literature [23], which might be associated with some small sample sizes' groups that could not be calculated cutoff-values.

However, some limitations in this study are as follows: Firstly, there were small sample sizes subgroups that have been excluded to calculate cutoff-values, so that we could not properly compare the influence of different risk factors on troponin values. Secondly, we did not include the particular of uremia patients with minority. Thirdly, there were other hs-cTn assays, but in this study, we only applied hs-TnI assay to comment on the clinical utility in CKD individuals. Fourthly, we just performed subgroups analysis according to age, gender and renal function because of the limitation of sample size, but there were other related factors that could influence hs-cTn concentration, such as Type 2 diabetes, Hypertension, Neuromuscular diseases and so on $[9,24,25]$. New studies including larger sample size should be performed and grouped by more factors as mentioned above. Fifthly, we could not compare the optimal cut-off values according to different age stages. Lastly, this retrospective study was conducted based on medical records from a single center. There should be more multicenter prospective randomized controlled trials to evidence.

\section{Conclusion}

Different optimal cutoff-values of hs-cTnI for diagnosis of AMI in CKD patients were useful for clinical diagnosis of AMI in various populations and were higher in males 
than females, and 7 of 9 subgroups' specificity reached to $100 \%$, but which were needed to be validated by multicenter randomized controlled clinical studies in the future.

\begin{abstract}
Abbreviations
AMI: Acute myocardial infarction; CKD: Chronic kidney disease; eGFR: Estimated glomerular filtration rate; CAD: Coronary artery disease; Hs-cTnl: Highsensitive cardiac troponin I; Cl: Confidence interval; AUC: Area under the curve; ROC: Receiver-operating characteristic; ACS: Acute Coronary Syndrome; CAG : Coronary angiography; CVD: Cardiovascular disease; CM: Contrast media; $\mathrm{Cl}$ AKI: Contrast induced-acute kidney injury; DM: Diabetes mellitus; SBP: Systolic blood pressure; DBP: Diastolic blood pressure.
\end{abstract}

\section{Acknowledgments \\ Not applicable.}

\section{Authors' contributions}

DR collected the data, reviewed articles, analyzed data and wrote the manuscript. TH and XL helped complete the data analysis. GX designed the study and revised the manuscript. All the authors approved the final version of the manuscript.

\section{Funding}

This work was supported by the National Natural Science Foundation of China (No. 81970583) and the Nature Science Foundation of Jiangxi Province (No. 20181BAB205016).

\section{Availability of data and materials}

The datasets generated during the current study are not publicly available because which involves confidential patient data but are available from the corresponding author on reasonable request.

\section{Ethics approval and consent to participate}

The study was carried out in accordance with the application principle of the Second Affiliated Hospital of Nanchang University, and all experimental schemes were approved by the hospital's Ethics Committee. The approval number was Review [2011] No. (001). Because the study was retrospective, informed consent was not required.

\section{Consent for publication}

Not applicable.

\section{Competing interests}

Authors stated no conflict of interest.

Received: 15 July 2020 Accepted: 19 October 2020

Published online: 17 February 2021

\section{References}

1. Sarnak MJ, Levey AS, Schoolwerth AC, et al. Kidney disease as a risk factor for development of cardiovascular disease: a statement from the American Heart Association councils on kidney in cardiovascular disease, high blood pressure research, clinical cardiology, and epidemiology and prevention. Circulation. 2015;108:2154-69.

2. Fox CS, Muntner P, Chen AY, et al. Acute Coronary Treatment and Intervention Outcomes Network Registry. Use of evidence-based therapies in shortterm outcomes of ST-segment elevation myocardial infarction and non-STsegment elevation myocardial infarction in patients with chronic kidney disease: a report from the National Cardiovascular Data Acute Coronary Treatment and Intervention Outcomes Network registry. Circulation. 2010;121:357-65. https://doi.org/10.1161/CIRCULATIO NAHA. 109.865352.

3. Wybraniec MT, Bożentowicz-Wikarek M, Chudek J, et al. Pre-procedural renal resistive index accurately predicts contrast-induced acute kidney injury in patients with preserved renal function submitted to coronary angiography. Int J Cardiovasc Imaging. 2017;33(5):595-604. https://doi. org/10.1007/s10554-016-1039-1.

4. Huang HL, Zhu S, Wang WQ, et al. Diagnosis of acute myocardial infarction in patients with renal insufficiency using high-sensitivity troponin $\mathrm{T}$. Clin Chem Lab Med. 2015;53(5):723-30.

5. Anavekar NS, McMurray JJ, Velazquez EJ, et al. Relation between renal dysfunction and cardiovascular outcomes after myocardial infarction. N Engl J Med. 2004;351(13):1285-95. https://doi.org/10.1056/NEJMoa0413 65.

6. Roffi M, Patrono C, Collet J-P, et al. 2015 ESC guidelines for the management of acute coronary syndromes in patients presenting without persistent ST-segment elevation: Task Force for the management of acute coronary syndromes in patients presenting without persistent STsegment elevation of the European Society of Cardiology (ESC). Eur Heart J. 2016;37:267-315.

7. Thygesen K, Alpert JS, Jaffe AS, et al. Fourth universal definition of myocardial infarction. Eur Heart J. 2018;00:1-33. https://doi.org/10.1093/ eurheartj/ehy462.

8. Monneret D, Gellerstedt M, Bonnefont-Rousselot D. Determination of age- and sex-specific 99th percentiles for high-sensitive troponin T from patients: an analytical imprecision- and partitioning-based approach. Clin Chem Lab Med. 2018;56(5):685-96.

9. Hallen J, Johansen OE, Birkeland Kl, et al. Determinants and prognostic implications of cardiac troponin T measured by a sensitive assay in type 2 diabetes mellitus. Cardiovasc Diabetol. 2010;9:52.

10. Twerenbold R, Badertscher P, Boeddinghaus J, et al. 0/1-hour triage algorithm for myocardial infarction in patients with renal dysfunction. Circulation. 2018;137:436-51. https://doi.org/10.1161/CIRCULATIO NAHA.117.028901.

11. Keller T, Zeller T, Ojeda F, et al. Serial changes in highly sensitive troponin I assay and early diagnosis of myocardial infarction. AMA. 2011;306:2684-93.

12. Roger VL, Go AS, Lloyd-Jones DM, et al. Heart disease and stroke statistics-2012 update: a report from the American Heart Association. Circulation. 2012;125(1):e2-220. https://doi.org/10.1161/CIR.0b013e31823ac046.

13. Twerenbold R, Wildi K, Jaeger C, et al. Optimal cutoff levels of more sensitive cardiac troponin assays for the early diagnosis of myocardial infarction in patients with renal dysfunction. Circulation. 2015;131(23):2041-50. https://doi.org/10.1161/CIRCULATIONAHA.114.014245.

14. Levin A, KdlgoKCW Group. KDIGO 2012 clinical practice guideline for the evaluation and management of chronic kidney disease. Kidney Int Suppl. 2013:3:1-150.

15. Jaffe AS. Chasing troponin: how low can you go if you can see the rise? Am Coll Cardiol. 2006:48:763-1764.

16. Wong JA, Goodman SG, Yan RT, et al. Canadian Acute Coronary Syndromes I and II, and Canadian Global Registry of Acute Coronary Events (GRACE/GRACE) Investigators. Temporal management patterns and outcomes of non-ST elevation acute coronary syndromes in patients with kidney dysfunction. Eur Heart J. 2009;30:549-57.

17. Shroff GR, Frederick PD, Herzog CA. Renal failure and acute myocardial infarction: clinical characteristics in patients with advanced chronic kidney disease, on dialysis, and without chronic kidney disease. A collaborative project of the United States Renal Data System/National Institutes of Health and the National Registry of Myocardial Infarction. Am Heart J. 2012;163:399-406.

18. Kraus D, von Jeinsen B, Tzikas S, et al. Cardiac troponins for the diagnosis of acute myocardial infarction in chronic kidney disease. Am Heart Assoc. 2018;7:e008032. https://doi.org/10.1161/JAHA.117.008032.

19. Yang HL, Liu J, Luo H, et al. Improving the diagnostic accuracy of acute myocardial infarction with the use of high-sensitive cardiac troponin $\mathrm{T}$ in different chronic kidney disease stages. Sci Rep. 2017;7:41350. https://doi. org/10.1038/srep41350.

20. Schulz O, Paul-Walter C, Lehmann M, et al. Usefulness of detectable levels of troponin, below the 99th percentile of the normal range, as a clue to the presence of underlying coronary artery disease. Am J Cardiol. 2007:100(5):764-9.

21. de Lemos JA, Drazner MH, Omland T, et al. Association of troponin T detected with a highly sensitive assay and cardiac structure and mortality risk in the general population. JAMA. 2010;304(22):2503-12. 
22. Irfan A, Reichlin T, Twerenbold R, et al. Early diagnosis of myocardial infarction using absolute and relative changes in cardiac troponin concentrations. Am J Med. 2013;126(9):781-788.e782

23. Shah AS, Griffiths M, Lee KK, et al. High sensitivity cardiac troponin and the under-diagnosis of myocardial infarction in women: prospective cohort study. BMJ (Clin Res Ed). 2015;350:g7873.

24. Jaffe AS, Vasile VC, Milone M, et al. Diseased skeletal muscle: a noncardiac source of increased circulating concentrations of cardiac troponin T. J Am Coll Cardiol. 2011;58(17):1819-24.
25. Eggers KM, Lindahl B. Application of cardiac troponin in cardiovascular diseases other than acute coronary syndrome. Clin Chem. 2017;63(1):223-35.

\section{Publisher's Note}

Springer Nature remains neutral with regard to jurisdictional claims in published maps and institutional affiliations.
Ready to submit your research? Choose BMC and benefit from:

- fast, convenient online submission

- thorough peer review by experienced researchers in your field

- rapid publication on acceptance

- support for research data, including large and complex data types

- gold Open Access which fosters wider collaboration and increased citations

- maximum visibility for your research: over $100 \mathrm{M}$ website views per year

At BMC, research is always in progress.

Learn more biomedcentral.com/submissions 